\title{
Stability and approximation of solutions in new reproducing kernel Hilbert spaces on a semi-infinite domain
}

\author{
Jabar Hassan ${ }^{1}$ and David E. Grow ${ }^{2}$ \\ ${ }^{1}$ Salahaddin University - Erbil College of Science \\ ${ }^{2}$ Missouri University of Science and Technology
}

March 3, 2021

\begin{abstract}
We introduce new reproducing kernel Hilbert spaces on a trapezoidal semi-infinite domain $\$ B_{-}\{\backslash$ infty $\} \$$ in the plane. We establish uniform approximation results in terms of the number of nodes on compact subsets of $\$ \mathrm{~B}_{-}\{\backslash$ infty $\} \$$ for solutions to nonhomogeneous hyperbolic partial differential equations in one of these spaces, $\$ \backslash$ widetilde $\{\mathrm{W}\}\left(\mathrm{B}_{-}\{\backslash\right.$ infty $\left.\}\right) \$$. Furthermore, we demonstrate the stability of such solutions with respect to the driver. Finally, we give an example to illustrate the efficiency and accuracy of our results.
\end{abstract}

\section{Hosted file}

Stability_and_Approximation.pdf available at https://authorea.com/users/399289/articles/ 511826-stability-and-approximation-of-solutions-in-new-reproducing-kernel-hilbertspaces-on-a-semi-infinite-domain 